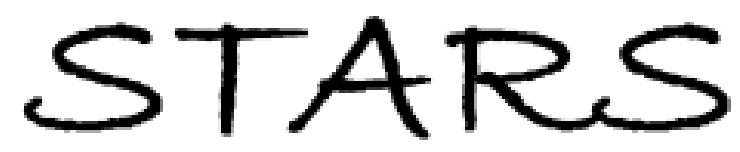

University of Central Florida

STARS

$1-1-2014$

\title{
Image sticking in liquid crystal displays with lateral electric fields
}

\author{
Daming $\mathrm{Xu}$ \\ University of Central Florida \\ Fenglin Peng \\ University of Central Florida \\ Haiwei Chen \\ University of Central Florida \\ Jiamin Yuan \\ University of Central Florida \\ Shin-Tson Wu \\ University of Central Florida
}

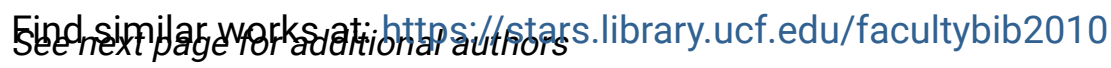

University of Central Florida Libraries http://library.ucf.edu

This Article is brought to you for free and open access by the Faculty Bibliography at STARS. It has been accepted for inclusion in Faculty Bibliography 2010 s by an authorized administrator of STARS. For more information, please contactSTARS@ucf.edu.

\section{Recommended Citation}

Xu, Daming; Peng, Fenglin; Chen, Haiwei; Yuan, Jiamin; Wu, Shin-Tson; Li, Ming-Chun; Lee, Seok-Lyul; and Tsai, Weng-Ching, "Image sticking in liquid crystal displays with lateral electric fields" (2014). Faculty Bibliography 2010s. 6312.

https://stars.library.ucf.edu/facultybib2010/6312

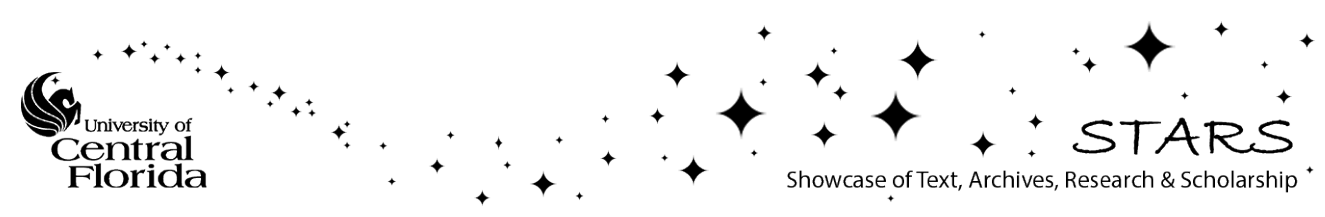




\section{Authors}

Daming Xu, Fenglin Peng, Haiwei Chen, Jiamin Yuan, Shin-Tson Wu, Ming-Chun Li, Seok-Lyul Lee, and Weng-Ching Tsai 


\section{Image sticking in liquid crystal displays with lateral electric fields}

Cite as: J. Appl. Phys. 116, 193102 (2014); https://doi.org/10.1063/1.4902083

Submitted: 13 October 2014 . Accepted: 07 November 2014 . Published Online: 20 November 2014

Daming Xu, Fenglin Peng, Haiwei Chen (D), Jiamin Yuan, Shin-Tson Wu (D), Ming-Chun Li, Seok-Lyul Lee, and Weng-Ching Tsai
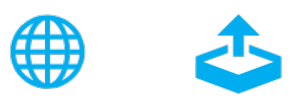

\section{ARTICLES YOU MAY BE INTERESTED IN}

Generation mechanism of residual direct current voltage in a liquid crystal display and its evaluation parameters related to liquid crystal and alignment layer materials

Journal of Applied Physics 102, 014904 (2007); https://doi.org/10.1063/1.2752147

Behavior of ion affecting image sticking on liquid crystal displays under application of direct current voltage

Journal of Applied Physics 108, 104903 (2010); https://doi.org/10.1063/1.3504186

Alignment layer effects on thin liquid crystal cells

Applied Physics Letters 92, 061102 (2008); https://doi.org/10.1063/1.2841642

\section{Applied Physics Reviews} Now accepting original research

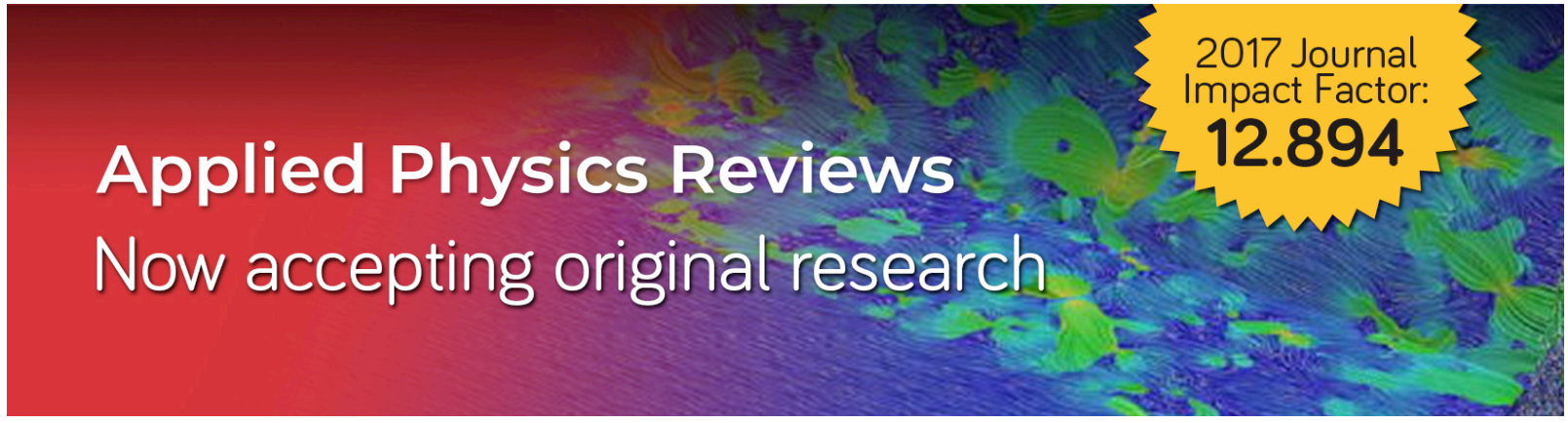




\title{
Image sticking in liquid crystal displays with lateral electric fields
}

\author{
Daming Xu, ${ }^{1}$ Fenglin Peng, ${ }^{1}$ Haiwei Chen, ${ }^{1}$ Jiamin Yuan, ${ }^{1}$ Shin-Tson Wu, ${ }^{1, a)}$ Ming-Chun Li, ${ }^{2}$ \\ Seok-Lyul Lee, ${ }^{2}$ and Weng-Ching Tsai ${ }^{2}$ \\ ${ }^{1}$ College of Optics and Photonics, University of Central Florida, Orlando, Florida 32816, USA \\ ${ }^{2}$ AU Optronics Corp., Hsinchu Science Park, Hsinchu 300, Taiwan
}

(Received 13 October 2014; accepted 7 November 2014; published online 20 November 2014)

\begin{abstract}
We propose a kinetic model to account for the nonuniform adsorption and desorption processes in fringe field switching (FFS) and in-plane-switching liquid crystal displays. An equation is proposed to describe the generation mechanism of residual DC voltage and good agreements with experiment are obtained. Based on this model, the mechanisms underlying the formation and relaxation processes of residual DC voltage as well as their dependences on offset DC voltage and temperature are investigated. Moreover, the residual DC voltages of FFS cells employing positive and negative dielectric anisotropy LCs are compared and the physics responsible for the observed difference is explained. (O 2014 AIP Publishing LLC. [http://dx.doi.org/10.1063/1.4902083]
\end{abstract}

\section{INTRODUCTION}

After half a century of tremendous efforts in material research and device development, followed by massive investment in advanced manufacturing technology, thin-film transistor liquid crystal display (TFT LCD) has become the mainstream flat panel display technology nowadays. ${ }^{1-3}$ Its widespread applications include smartphones, tablets, computer screens, and TVs. Nevertheless, the demand for better image quality is ever-increasing, such as wide viewing angle for multi-viewers, high resolution for Retina display, and pressure-resistance for touch screen. ${ }^{4}$ In-plane switching (IPS) $)^{5-7}$ and fringe field switching (FFS $)^{8-10}$ modes, in which the electric-field-induced LC molecular reorientation takes place mainly in the lateral direction, satisfy above criteria and are commonly used in mobile displays and high-end LCDs. ${ }^{11}$

However, some technical issues still remain to be solved for these two modes, such as slow response time especially at low temperature, ${ }^{12}$ and image sticking (also referred to as burn-in effect or ghosting). ${ }^{13-16}$ Image sticking is a phenomenon that a faint outline of previously displayed image remains visible on the screen as the frame is refreshed. It arises from ionic charges accumulated at interface between liquid crystals (LCs) and alignment layer (hereinafter referred to as "interface") when the display panel has been operated continuously for a long period of time with a fixed image. When the external driving voltage is removed, the ions do not dissipate from the interface immediately which in turn gives rise to a residual direct current (DC) voltage, resulting in a retention of displayed image. Image sticking is annoying to users as it degrades the image quality ${ }^{17}$ and should be minimized or eliminated.

To clarify the correlation between ion activities and residual DC voltage, some earlier studies have been reported. Yasuda et al. attributed the occurrence of residual DC voltage to ion adsorption and desorption; they observed these processes experimentally and built a physical model to describe them. ${ }^{18}$ Afterwards, many efforts were devoted to

${ }^{a)}$ Electronic mail: swu@ucf.edu explore the physical mechanisms behind the adsorption and desorption processes, both experimentally and theoretically. ${ }^{19-24}$ Recently, based on Yasuda's model Mizusaki et $a l .{ }^{24}$ proposed a more comprehensive kinetic model to evaluate the ion adsorption and desorption processes in homogeneous LC cells. This model is based on the assumption that ion adsorption is uniform over the entire interface under uniform longitudinal electric field, thus it does not apply to FFS or IPS cell, in which the electric field is in lateral direction and the field distribution is not uniform. Hence, we need to develop a new model to describe the generation mechanisms of residual DC voltage in these modes with nonuniform lateral fields.

In this paper, we propose a kinetic model to characterize the nonuniform adsorption and desorption processes of FFS and IPS LCDs with lateral fields. Based on this model, the generation and relaxation mechanisms of residual DC voltage as well as the dependence of residual DC voltage on applied voltage and temperature are studied. Finally, the residual DC voltage in FFS cells employing positive and negative dielectric anisotropy $(\Delta \varepsilon)$ LCs are compared and the underlying physical mechanisms are explained.

\section{PHYSICAL MODEL}

\section{A. Generation mechanisms}

The first cause of residual DC voltage is the offset DC voltage originated from the swing of TFT drivers. Figure 1 illustrates the electrical model of a single LCD pixel. Due to the clock feedthrough effect, a decrement in the voltage applied to the LC cell $\left(V_{L C}\right)$ would be generated when the TFT is turned OFF at the end of a selected period. Assuming $\Delta V_{G}$ is the voltage change at the TFT gate when the row is deselected, then the voltage shift of $V_{L C}$ is expressed as ${ }^{25}$

$$
\Delta V_{L C}=\frac{C_{G D}}{C_{G D}+C_{S}+C_{L C}} \cdot \Delta V_{G},
$$

where $C_{G D}$ is the gate-data parasitic capacitance of a TFT, $C_{S}$ is the storage capacitance and $C_{L C}$ is the LC capacitance. 


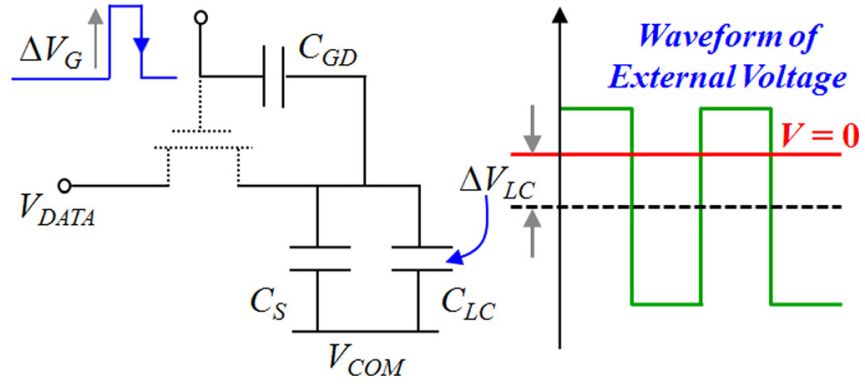

FIG. 1. Pixel electrical model for the generation of offset DC voltage.

The voltage shift $\Delta V_{L C}$ would generate an unwanted DC component and cause a modification of the alternating current $(\mathrm{AC})$ data signal: this DC voltage adds to the applied voltage in one frame, say negative frame, and decreases the applied voltage in the positive frame, thus generating a net voltage difference between positive and negative frames, as shown in Fig. 1. This net voltage difference would render the free ions drift toward the interface during long-time driving. Consequently, the adsorbed ions would generate a residual DC voltage, which persists even after the external voltage is removed, causing image sticking.

Meanwhile, polar LC compounds are asymmetric so that splay or bend deformation of the LC directors would give rise to an electric polarization. This feature was first discovered by Meyer in 1969 and is known as flexoelectric effect. $^{26}$ For example, in a FFS cell large splay and bend deformations are generated at the edges and between the pixel electrodes, respectively; especially for a FFS cell employing a positive $\Delta \varepsilon$ LC material (p-FFS). ${ }^{9,12}$ And these deformations would induce a flexoelectric polarization $\vec{P}$, which can be written as ${ }^{26}$

$$
\vec{P}=e_{1}(\vec{n} \nabla \cdot \vec{n})+e_{3}(\vec{n} \times \nabla \times \vec{n}),
$$

where $e_{1}$ and $e_{3}$ are the splay and bend flexoelectric coefficients and $\vec{n}$ is the unit vector of LC directors. The flexoelectric polarization introduces an additional term in the Gibbs free energy and causes some modifications in the Freedericksz transition. ${ }^{15,27}$ Therefore, the actual LC distribution profiles for positive and negative frames would be different although the device is driven by a pure AC signal, resulting in different voltage-transmittance (VT) curves. ${ }^{15,28}$ Subsequently, a net DC voltage is generated and ions are accumulated at the interface, giving rise to image sticking and flickering problems.

\section{B. Adsorption and desorption processes}

Before the occurrence of an offset DC voltage, the free ions are uniformly distributed in the LC layer. Upon the presence of an offset DC voltage, these free ions would drift toward the interface. ${ }^{18}$ When an LCD has been driven for a long period of time (usually $\sim 10$ min to several hours), the ions are trapped at the interface, generating a residual DC voltage. Mizusaki et al. found that the density of adsorbed ions are proportional to the electric field intensity in a homogeneous cell. ${ }^{24}$ However, compared to LC cells with uniform field, the scenario of FFS mode is much more

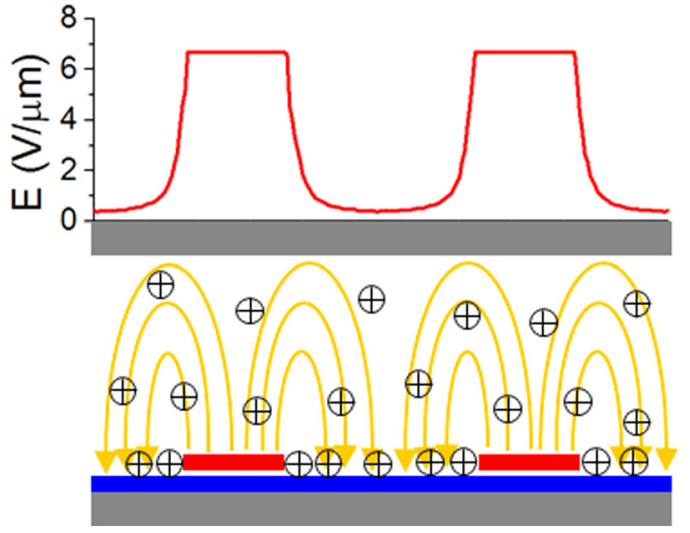

FIG. 2. Nonuniform electric field distribution and ion adsorption in a FFS-2/ 3.5 cell at $1 \mathrm{~V}$ DC voltage.

complicated as its electric field profile is not uniform. Figure 2 illustrates the field distribution of a FFS-2/3.5 cell (electrode width $W=2 \mu \mathrm{m}$, electrode gap $G=3.5 \mu \mathrm{m}$ ) when an external DC voltage of $1 \mathrm{~V}$ is applied. As a result, the distribution of adsorbed ions is position-dependent in a FFS cell. ${ }^{15}$ Murakami and Naito ${ }^{29}$ found that the mobile ionic charges in LCs were positive through measuring the transient photocurrent. Hence, only positive ions are shown in Fig. 2 to illustrate the adsorption mechanism.

In order to account for the nonuniform field in a FFS cell, we first assume the adsorbed ion density under a unit electric field is $n_{0}(t)$. When a unit electric field is applied, ions drift toward and are adsorbed to the interface. However, adsorption is not the only process that takes place during this period; its reverse process, desorption occurs as well. As time increases, more ions are adsorbed to the interface, but in the meantime the desorption process becomes more intense as well. Thus, the adsorbed ion density reaches saturation when an equilibrium state is achieved between the adsorption and desorption processes. Following rate equation can be used to describe the time-dependent adsorbed ion density under a unit electric field:

$$
d n_{0}(t) / d t=R_{a} \cdot\left[n_{s}-n_{0}(t)\right]-R_{d} \cdot n_{0}(t),
$$

where $n_{s}$ is the density of free ions existing in the LC around the interface and $R_{a}$ and $R_{d}$ are adsorption and desorption rate constants, respectively. Equation (3) has following solution:

$$
n_{0}(t)=\frac{1}{R_{a}+R_{d}}\left[R_{a} \cdot n_{s}-C \cdot e^{-\left(R_{a}+R_{d}\right) t}\right],
$$

where $C$ is a constant. By take the initial condition $n_{0}(0)=0$ into consideration, we find $C=R_{a} \cdot n_{s}$. Therefore, we can rewrite $n_{0}(t)$ as

$$
n_{0}(t)=\frac{R_{a} \cdot n_{s}}{R_{a}+R_{d}}\left[1-e^{-\left(R_{a}+R_{d}\right) t}\right] .
$$

Since the density of adsorbed ions is proportional to the applied electric field, the position-dependent adsorption under nonuniform electric field in a FFS cell can be written as 


$$
n(x, t)=\frac{E(x)}{1 \mathrm{~V} / \mu m} n_{0}(t),
$$

where $E(x)$ is the intensity of position-dependent electrical field, as Fig. 2 depicts. Therefore, the average adsorbed ion density $n_{a}(t)$ over the whole adsorption region can be calculated through

$$
n_{a}(t)=\langle n(x, t)\rangle=\langle E(x)\rangle \cdot n_{0}(t) .
$$

Here, $\langle E(x)\rangle$ is the average electrical field intensity over the adsorption region. The relationship between the residual DC voltage $V_{r}$ and $n_{a}$ is as follows:

$$
Q(t)=q \cdot n_{a}(t)=C_{L C} \cdot V_{r}(t),
$$

where $Q(t)$ is the surface electric charge, $C_{L C}$ is the LC capacitance, and $q$ is a constant $\left(1.6 \times 10^{-19} \mathrm{C}\right)$. Hence, we obtain following expression for $V_{r}$ :

$$
V_{r}(t)=\frac{q}{C_{L C}} \cdot \frac{R_{a} \cdot n_{s}}{R_{a}+R_{d}}\left[1-e^{-\left(R_{a}+R_{d}\right) t}\right] \cdot\langle E(x)\rangle .
$$

The exponential term of Eq. (9) implies that $V_{r}(t)$ would reach a saturation level after a sufficiently long period $(t \gg$ $\left.\left(R_{a}+R_{d}\right)^{-1}\right)$. Therefore, to evaluate $V_{r}(t)$ as a function of time, Eq. (9) can be simplified as

$$
V_{r}(t)=V_{r, S}\left[1-e^{-\left(R_{a}+R_{d}\right) t}\right],
$$

where $V_{r, s}$ is the saturated residual DC voltage. Meanwhile, the second term in Eq. (9) mainly depends on the properties of LC and alignment materials employed and needs to be investigated through experiments, which will be outlined later. However, the third term $\langle E(x)\rangle$ is determined by the device parameters and can be calculated through simulations. We simulated the electric field distribution of FFS and IPS cells using a commercial simulator TechWiz LCD (Sanayi System Co., Korea). Figure 3 shows the $\langle E(x)\rangle$ over the adsorption regions in FFS and IPS cells with different ratios between electrode width $(W)$ and electrode gap $(G)$ under an applied voltage of $1 \mathrm{~V}$. The $G / W$ ratio increases from 0.5 to 3 , which covers the commonly employed electrode configurations in

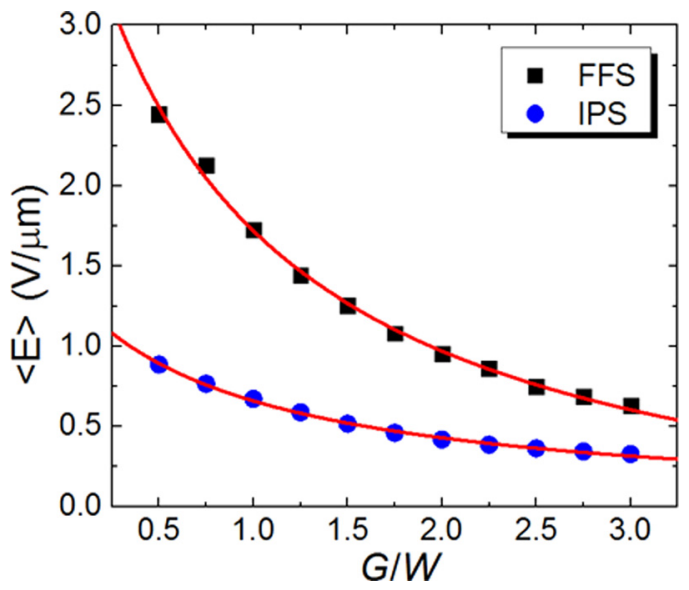

FIG. 3. Simulated $\langle\mathrm{E}(\mathrm{x})\rangle$ (dots) over the adsorption region for FFS and IPS cells under $1 \mathrm{~V}$ applied voltage. Solid lines are fittings with Eq. (11). the FFS and IPS structures. As the $G / W$ ratio increases, $\langle E(x)\rangle$ decreases dramatically. And this trend can be fitted by following equations:

$$
\begin{aligned}
& \text { FFS : }\langle E(x)\rangle=\frac{2.979-0.369 \cdot G / W}{0.727+0.791 \cdot G / W}, \\
& \text { IPS : }\langle E(x)\rangle=\frac{1.536-0.025 \cdot G / W}{1.119+1.171 \cdot G / W} .
\end{aligned}
$$

Therefore, when we plug Eq. (11) into Eq. (9) we can obtain a universal equation describing the nonuniform adsorption in a FFS or IPS cell. Next step is to investigate the properties of $R_{a}$ and $R_{d}$ by measuring the time-dependent $V_{r}$.

\section{EXPERIMENT}

\section{A. Measurement method}

To determine $V_{\mathrm{r}}$, both electrical and optical measurements have been proposed. ${ }^{24,30-32}$ Among them, electrical measurement method is more accurate as it measures $V_{r}$ directly, whereas the optical method needs to convert the optical transmittance change into $V_{r}$.

Figure 4 illustrates the electrical schematic of a circuit used for measuring $V_{\mathrm{r}}$. First, we apply a voltage $V_{a}$ on the LC cell for time $t_{1}$ (usually $\sim 10 \mathrm{~min}$ to several hours) by closing switch SW1 and opening SW2. During this process, ions are adsorbed to the interface. Then, we open SW1 and close SW2 for a short duration $t_{2}$ for discharging purpose. The discharge time is usually around several seconds, long enough for free ions to move but too short for adsorbed ones to escape the interface. In order to maintain a zero voltage at both substrates, some free charges must remain on the electrodes to balance the electric field generated by the adsorbed ions. After that, we open both SW1 and SW2 and measure $V_{s}$ for time $t_{3}$, which can vary from a few seconds to $10 \mathrm{~h}$. During this process, the adsorbed ions are slowly released from the interface and $V_{s}$ increases from $0 \mathrm{~V}$ to a maximum voltage, as the balancing effect of the adsorbed ions is slowly lost. Finally, $V_{s}$ will reach a saturation level. Note here that the key issue for an accurate measurement is to have an extremely small leakage current from the measurement circuit (e.g., <50 fA). In our experiments, we used ALCTE (Instec, USA) to measure the residual DC voltage.

\section{B. Generation process of residual DC voltage}

To understand the generation mechanism of residual DC voltage in a FFS cell, we prepared two different FFS cells by

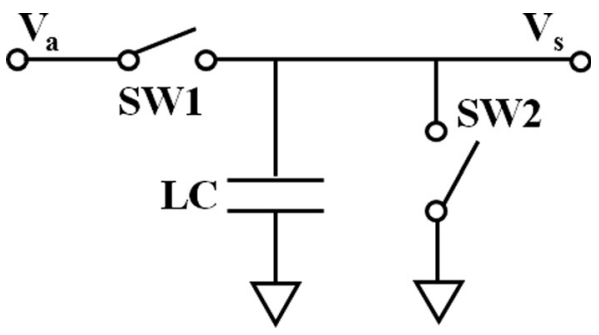

FIG. 4. Electrical schematic of the circuit used for measuring residual DC voltage. 
TABLE I. Physical properties of two LC mixtures studied $\left(\mathrm{T}=23^{\circ} \mathrm{C}\right.$, $\lambda=633 \mathrm{~nm}$ and $\mathrm{f}=1 \mathrm{kHz}$ ).

\begin{tabular}{lccccc}
\hline \hline LC & $T_{n i}\left({ }^{\circ} \mathrm{C}\right)$ & $\varepsilon_{/ /}$ & $\varepsilon_{\perp}$ & $\Delta \varepsilon$ & $\Delta \mathrm{n}$ \\
\hline MLC-6686 & 71.0 & 14.5 & 4.5 & 10.0 & 0.0969 \\
MLC-6882 & 69.0 & 3.6 & 6.7 & -3.1 & 0.0976 \\
\hline \hline
\end{tabular}

injecting Merck MLC-6686 into a positive FFS (p-FFS) cell and MLC-6882 into a negative FFS (n-FFS) cell. The physical properties of these two mixtures are listed in Table I. The cell parameters are as follows: electrode width $W=2 \mu \mathrm{m}$, electrode gap $G=3.5 \mu \mathrm{m}$, cell gap $\mathrm{d} \sim 3.2 \mu \mathrm{m}$, and the thickness of ITO electrodes is $50 \mathrm{~nm}$. Both cells are with photo-alignment $^{33}$ and the dielectric constant of photo-alignment layer is $\varepsilon=3.9$. The passivation layer between pixel and common electrode is $\mathrm{Si}_{3} \mathrm{~N}_{4}\left(\varepsilon_{p}=6.5\right)$ with a thickness $d_{p}=400 \mathrm{~nm}$. During measurement, $V_{\mathrm{r}}$ was evaluated after applying an external DC voltage for different time periods, increasing from $10 \mathrm{~min}, 20 \mathrm{~min}, 30 \mathrm{~min}$, to $1 \mathrm{~h}, 2 \mathrm{~h}, 3 \mathrm{~h}$, and $4 \mathrm{~h}$.

\section{Voltage effect}

We first investigated the voltage effect by measuring the time-dependent $V_{r}$ curves under different applied voltages at $23^{\circ} \mathrm{C}$, as represented by the discrete points in Fig. 5(a). Due to the limitation of space, here we only show the measured
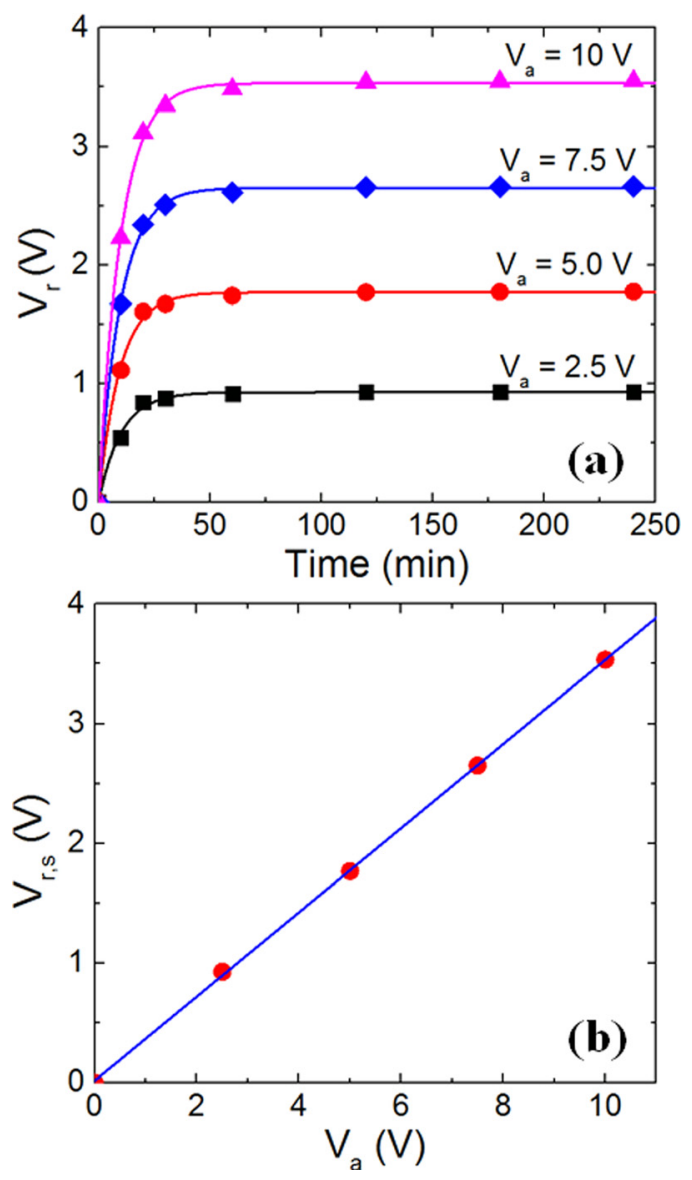

FIG. 5. (a) $V_{r}$ of the p-FFS cell as a function of time under different offset DC voltages at $23^{\circ}$ C. (b) Saturated residual DC voltage $V_{r, s}$ as a function of applied DC voltage. results of p-FFS cell, as the n-FFS cell exhibits the same trend. And the differences between n-FFS and p-FFS measurement results will be discussed later. It clearly shows that as time increases, $V_{r}$ gradually increases and finally saturates when the external DC voltage is applied for a sufficiently long period of time. We used Eq. (10) to fit the experimental results and the fitting curves are shown as solid lines in Fig. 5(a). The good agreement between fitting curves and experimental results indicates that our model well describes the generation process of $V_{r}$. Meanwhile, through fittings we obtained saturated residual DC voltage $V_{r, s}$ under different applied voltages, as plotted in Fig. 5(b). $V_{r, s}$ is clearly shown to be linearly proportional to $V_{a}$; this is because more ions are adsorbed to the interface under a higher voltage. ${ }^{24,34}$ Hence, our experimental results prove again that absorbed ion density is proportional to the intensity of electric field in a FFS cell, which validates our assumption in the model and shows same trend as a homogeneous cell. Note that $V_{r, s}$ directly determines the severity of image sticking, so it is crucial to reduce $V_{r, s}$ by optimizing the LC and alignment material properties. ${ }^{20,35,36}$

\section{Temperature effect}

In addition to the above voltage effect, we also investigated the temperature dependence of $V_{r}$. We measured the generation process of $V_{r}$ by applying a $5 \mathrm{~V}$ offset DC voltage to the p-FFS cell under different temperatures, increasing from $23,30,40,50$ to $55^{\circ} \mathrm{C}$, which is still lower than the clearing point of MLC-6686. The measured data are plotted as dots in Fig. 6 and the solid lines represent the fitting curves using Eq. (10).

Three trends are clearly shown in Fig. 6. First, Eq. (10) fits well with experimental data under all measured temperatures, showing that our model is suitable for describing the generation mechanism of $V_{r}$ under different temperatures. Second, as temperature increases, the saturated residual DC voltage increases as well. The fitted $V_{r, s}$ is linearly proportional to the reciprocal of temperature, as depicted in the inset plot in Fig. 6. This originates from that the free ion concentration in LC increases at an elevated temperature since more neutral impurities become ionized as the temperature

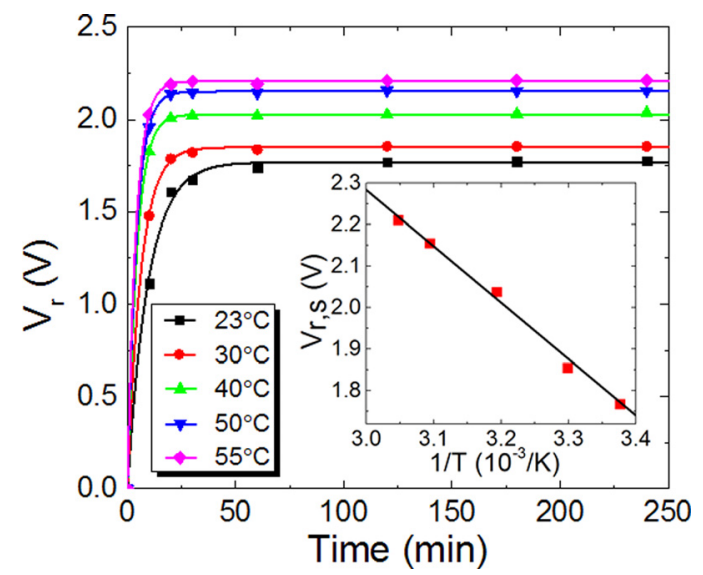

FIG. 6. Temperature dependence of $V_{r}$ (applied DC voltage: $5 \mathrm{~V}$ ). The inset plot shows the fitted $V_{r, s}$ under different temperatures. 
increases. $^{29}$ Thus, more ions are adsorbed to the interface and contribute to a higher $V_{r}$. Third, the slope of solid fitting curve increases as the temperature increases, showing that the exponent $R_{a}+R_{d}$ increases with temperature. This is because ions become more active at higher temperatures. To investigate the temperature dependence of ions adsorption and desorption processes, we evaluated $R_{a}$ and $R_{d}$ separately by using Eq. (9) to fit the measured data, and the fitting results are depicted as squares in Fig. 7. We can see that both $R_{a}$ and $R_{d}$ show concave-up curvatures, indicating that the adsorption and desorption of ions at the interface follow the Boltzmann distribution. ${ }^{37}$ This trend arises from the fact that both adsorption and desorption processes depend on the thermal motion of ions existing in the LC layer around the interface. Therefore, we used the Arrhenius equation to fit the $R_{a}$ and $R_{d}$ values

$$
R=R_{0} \exp (-E / k T)
$$

where $R_{0}$ is a rate constant, $E$ is the activation energy of adsorption or desorption processes, $k$ is the Boltzmann constant, and $T$ is the temperature. The activation energy of adsorption is considered as the energy required to overcome electric repulsion governed by the Coulomb interaction between the ions in the LC layer and the adsorbed ions on the interface. In contrast, the activation energy of desorption is the required energy for ions to get over the van der Waals interaction between ions and alignment layer. Through fittings, we obtained the activation energy $E_{a}=0.487 \mathrm{eV}$ for adsorption and $E_{d}=0.348 \mathrm{eV}$ for desorption, which are on the same order of magnitude with the activation energy of ions at the interface between polyimide and $5 \mathrm{CB}(\sim 0.5 \mathrm{eV}){ }^{29,38}$

\section{Relaxation process of residual DC voltage}

When the external DC voltage is removed after being applied for a long time, the adsorbed ions escape from the interface slowly, and this process usually takes several seconds to tens of minutes to finish. In our experiments, the relaxation process of $V_{r}$ was evaluated by measuring the temporal response of $V_{s}$. As defined above, $V_{s}$ is a voltage generated by the free charges remained on the electrodes to

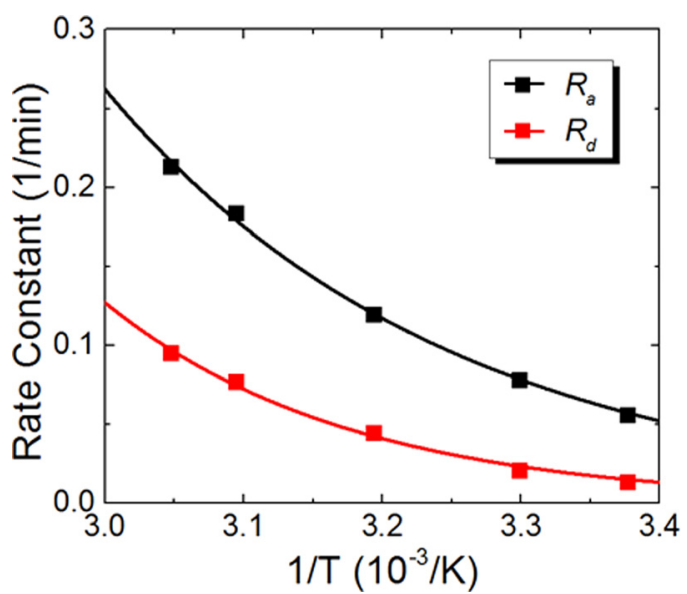

FIG. 7. Fitted adsorption and desorption rates (dots) under different temperatures. Solid lines represent the fitting curves with Eq. (12). balance the electric field generated by $V_{r}$. Therefore, $V_{s}$ is complementary to $V_{r}$ and is related to $V_{r}$ as

$$
V_{s}(t)+V_{r}(t)=V_{s, r}
$$

The black solid line in Fig. 8 shows the measured transient $V_{s}$ change of the p-FFS cell during the relaxation process after applying a $5 \mathrm{~V}$ external DC voltage for 4 hours at $23^{\circ} \mathrm{C}$. To further understand the physical mechanism underlying the transient $V_{s}$ change process, we used exponential equations to fit the measured data. We first started with a single exponential equation; however, it does not fit the measured data well [blue solid line in Fig. 8], indicating that more than one relaxation mechanism take place. Indeed, ions with different motilities such as $\mathrm{Na}^{+}, \mathrm{Al}^{3+}, \mathrm{Ca}^{2+}$, and $\mathrm{K}^{+}$are commonly found in a LC mixture. ${ }^{39}$ Some studies have also been reported to prove that more than one type of ions co-exist in LCs. ${ }^{40-42}$ For example, Sawada et al. discovered that there are as many as five types of ions in 5CB in terms of ion mobility. ${ }^{19}$ Therefore, there are multiple relaxation processes going on simultaneously and the desorption rates of different ions are different, as it can also be clearly observed from Fig. 8 that the measured relaxation involves two processes: a fast one followed by a slow one. Therefore, we extended the fitting equation to double exponential equation as follows:

$$
V_{s}(t)=V_{r, s} \cdot\left[A \cdot e^{-t \cdot R_{d, f}}+(1-A) \cdot e^{-t \cdot R_{d, s}}\right],
$$

where $R_{d, f}$ and $R_{d, s}$ denote the rate constant of fast and slow desorption processes, respectively; while A represents the contribution of fast desorption process. The fitting results (red dashed lines) show that the double exponential equations well describes the dynamic desorption processes.

Based on this desorption model, we also studied the temperature dependent relaxation process of $V_{r}$ by measuring the transient change of $V_{s}$ under different temperatures. Results are depicted in Fig. 9. It clearly shows that the slope of the curves increases dramatically as the temperature increases. To investigate the temperature effect of desorption process, we fitted all these curves with Eq. (14) and obtained the fast and slow desorption rates under different temperatures [discrete points in Fig. 10]. Then we used Eq. (12) to fit

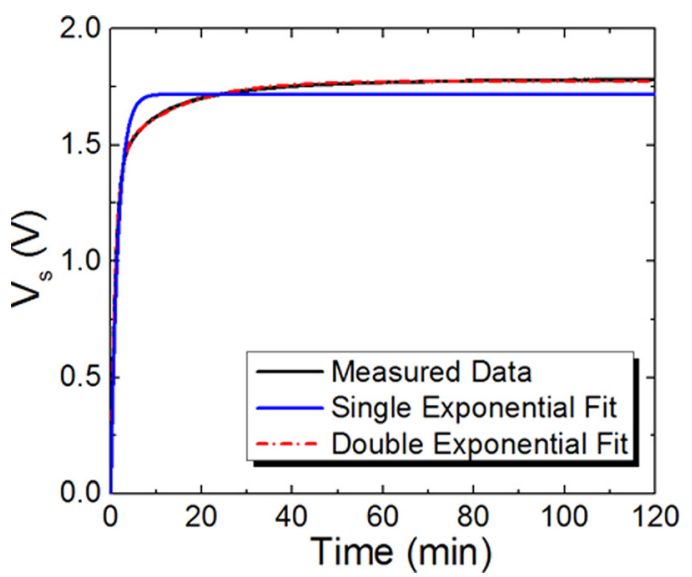

FIG. 8. Transient rise processes of $V_{s}$ of the p-FFS cell after applying a $5 \mathrm{~V}$ external DC voltage for $4 \mathrm{~h}$ at $23^{\circ} \mathrm{C}$. The red solid and blue dashed lines are fitting results using double and single exponential equations, respectively. 


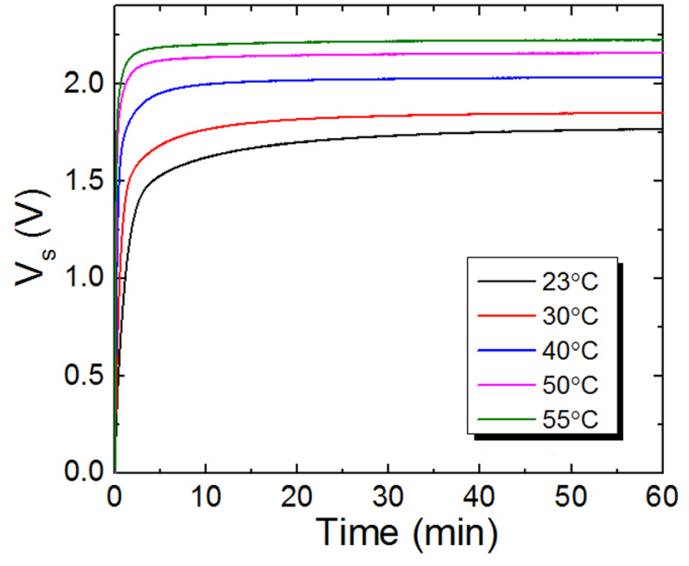

FIG. 9. Transient rise processes of $V_{s}$ of the p-FFS cell under different temperatures. The data were measured after applying a $5 \mathrm{~V}$ external $\mathrm{DC}$ voltage for $4 \mathrm{~h}$.

these points and found that the temperature dependence of fast and slow desorption processes follow the Arrhenius equation as well. The fitted activation energies for the fast and slow desorption processes are $0.271 \mathrm{eV}$ and $0.475 \mathrm{eV}$, respectively; showing that the adsorbed ions required a higher energy to escape the interface for the slow desorption process.

The different activation energies between fast and slow desorption processes result from the interaction between adsorbed ions and interface, which is mainly governed by the van der Waals force. ${ }^{18,41}$ It is widely known that the van der Waals force increases as the ion mass gets heavier. Therefore, heavier ions require a higher energy to overcome this force and escape from the interface. Accordingly, the ions with heavier mass are responsible for the slow desorption process, and vice versa. Roughly speaking, ions in a LC mixture can be divided into two groups: light and heavy. For example, Hung et al. $^{39}$ analyzed the metal ion impurities in several LC mixtures and reported the metal ion concentrations of a LCD panel with image sticking. Their results show that the metal ions in the image sticking region can be divided into two groups: heavy metal ions $(\mathrm{Ni}, \mathrm{Cu}, \mathrm{Zn}, \mathrm{Fe}$,

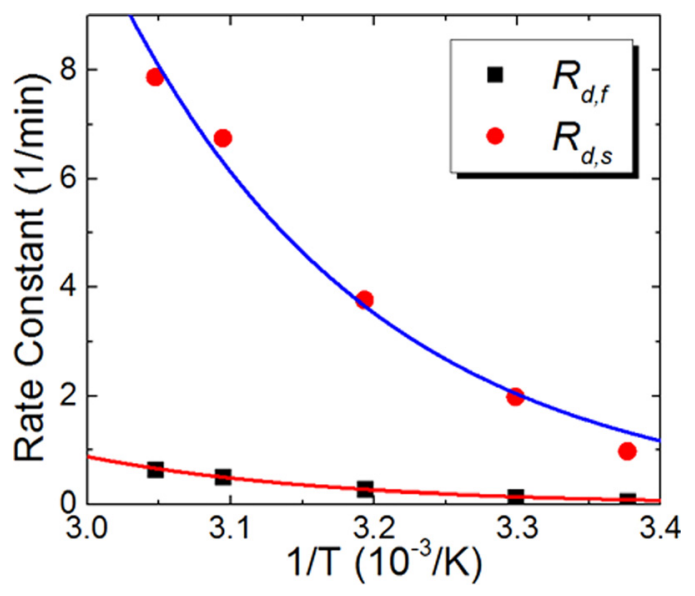

FIG. 10. Fitted rates for fast (black squares) and slow (red dots) desorption processes under different temperatures. Solid lines represent the fittings with Eq. (12).

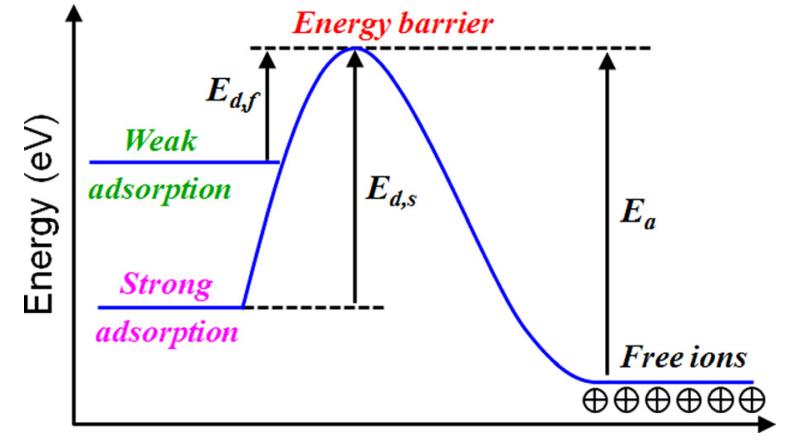

FIG. 11. Activation energy diagram for adsorption and desorption of ions.

etc.) with an average mass of 60.5 and lighter metal ions (Na, $\mathrm{Mg}, \mathrm{Al}, \mathrm{Ca}, \mathrm{K}$, etc.) with an average mass of 31.7. The total concentration of heavy metal ions are $\sim 24 \%$, which is quite close to our fitted ratio of the slow desorption process (18.6\%). Although the LC materials we employed are different from theirs, the ion constituents should not be very different and the trend should be similar as well. These ions are introduced during synthesis and are difficult to be completely removed during purification process.

In brief, the temperature dependence of adsorption and desorption processes were investigated and their activation energies are summarized and illustrated in Fig. 11. To adsorb the free ions in the LC layer onto the interface, an energy $E_{a}$ is required to overcome the Coulomb force between ions and climb over the energy barrier. Since the difference in ion mass results in different van der Waals interactions, weak and strong adsorptions can be differentiated. For strongly adsorbed ions, a higher energy $E_{d, s}$ is required to overcome the van der Waals force. Therefore, for the purpose of reducing image sticking, $\mathrm{LC}$ and alignment materials with a higher $E_{a}$ and lower $E_{d}$ are preferred. A higher $E_{a}$ means that the ions are more difficult to be adsorbed onto the interface and the severity of image sticking would be reduced. Meanwhile, a lower $E_{d}$ would render the adsorbed ions be released from the interface more easily and quickly, which means the image sticking would disappear faster after the removal of the offset DC voltage.

\section{D. p-FFS vs. $n$-FFS}

As mentioned above, due to the limitation of space, we only showed the results of p-FFS cell to illustrate the generation mechanisms of residual DC voltage because n-FFS exhibits same trends in voltage and temperature. However, there are still some differences between them due to the different LC properties, as outlined below.

Figure 12 shows the measured time-dependent $V_{r}$ curve of p-FFS and n-FFS at $23^{\circ} \mathrm{C}$ under an external DC offset voltage of $5 \mathrm{~V}$. As we can see here, n-FFS exhibits a higher $V_{r}$ than p-FFS, which means the image sticking is more severe for n-FFS. Moreover, we measured the time-dependent $V_{r}$ curve under different temperatures and obtained the adsorption and desorption rates through fitting by using the same method described above. The fitting results of p-FFS are shown as solid dots and squares in Fig. 13, while those of nFFS are represented by open diamonds and triangles. 


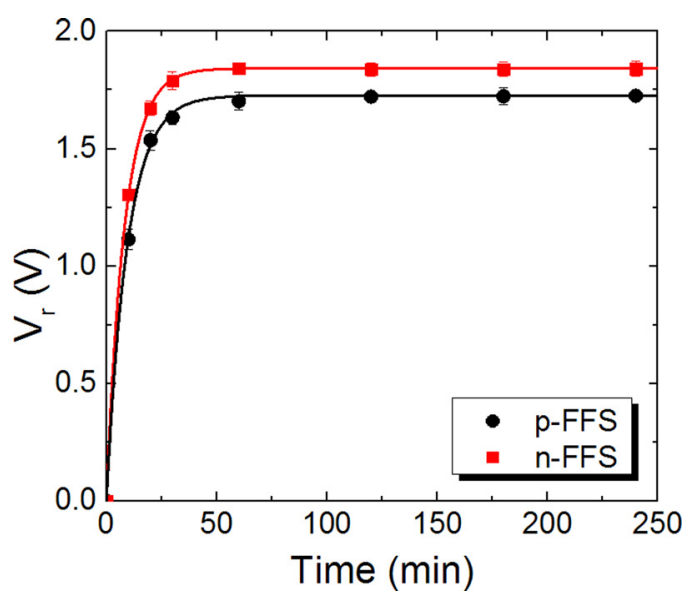

FIG. 12. Time-dependent $V_{r}$ curve of p-FFS and n-FFS. (Offset DC voltage $\left.V_{a}=5 \mathrm{~V}, 23^{\circ} \mathrm{C}\right)$.

Equation (12) was used to investigate the temperature dependence of these fitting results, and the fitted curves are plotted as solid lines in Fig. 13. Through fittings, we found that the adsorption activation energy of n-FFS is lower than that of $\mathrm{p}$ FFS $(0.425$ vs. $0.487 \mathrm{eV})$, but the desorption activation energy of n-FFS is higher $(0.412$ vs. $0.348 \mathrm{eV})$. This means in the nFFS cell it is easier for ions to be adsorbed to the interface but more difficult to desorb, resulting in a more severe image sticking.

Regarding these differences, we believe two reasons need to be considered. First, a negative $\Delta \varepsilon$ LC usually employs lateral difluoro compounds, which tend to contain more ionic impurities. Therefore, the free ion density in the negative LC is higher, resulting in a lower $\mathrm{VHR}^{16}$ and higher residual DC voltage higher for the n-FFS cell. Second, the mismatch between dielectric constant is also responsible for the more severe image sticking of $\mathrm{n}$-FFS. ${ }^{43}$ According to previous study, the change in free energy $(\Delta E)$ when an ion goes from a medium of dielectric constant $\varepsilon_{1}$ to another with $\varepsilon_{2}$ is ${ }^{44}$

$$
\Delta E=-\frac{Q^{2}}{8 \pi \varepsilon_{0} a}\left(\frac{1}{\varepsilon_{1}}-\frac{1}{\varepsilon_{2}}\right),
$$

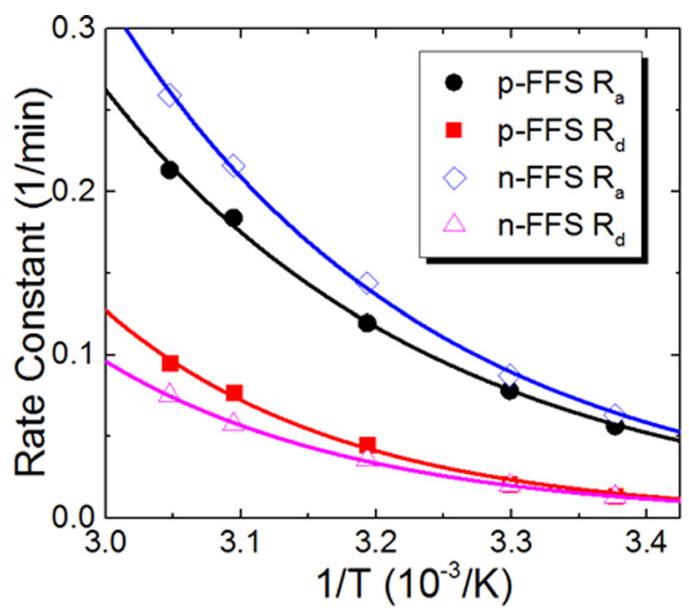

FIG. 13. Fitted adsorption and desorption rates of p-FFS (solid dots and squares) and n-FFS (open diamonds and triangles) under different temperatures. Solid lines represent the fitting curves with Eq. (12). where $Q$ is the charge of the ion and $a$ is the ion size. Therefore, it is energetically favorable to transfer ions from the LC layer to alignment layer with a smaller difference in dielectric constant. Here, the dielectric constant of photoalignment material is $\varepsilon=3.9$, while the average dielectric constant $\langle\varepsilon\rangle$ of the positive and negative LCs is 7.83 and 5.67, respectively. The smaller difference between dielectric constant of negative LC and alignment layer implies that ions require a lower energy to be adsorbed to interface, well matches with our fitting results. Hence, to minimize image sticking, an alignment layer with low $\varepsilon$ or a LC material with high $\langle\varepsilon\rangle$ is helpful.

\section{CONCLUSION}

We reported a kinetic model to account for the nonuniform adsorption and desorption processes in FFS and IPS cells. An equation was proposed to describe the generation mechanisms of residual DC voltage by taking the material properties and device structure parameters into consideration. Our model fits experimental results well and we studied the voltage and temperature effects of the residual DC voltage based on this model. For the adsorption process, an activation energy is required to overcome Coulomb repulsion force between ions in order to adsorb the free ions onto the interface. During the desorption process, ions can be categorized into two groups due to the difference in their mass and these two groups require different desorption activation energies and exhibit different desorption rates. In addition, the residual DC voltages of n-FFS and p-FFS cells are compared. Our results indicate that it is favorable to reduce the difference between the dielectric constants of LC and alignment layer. Our proposed model and experimental evidences are helpful to understand and minimize the image sticking of FFS and IPS LCDs.

\section{ACKNOWLEDGMENTS}

The authors are indebted to AU Optronics (AUO, Taiwan) and AFOSR for the financial supports under Contract No. FA9550-14-1-0279.

${ }^{1}$ M. Schadt, Annu. Rev. Mater. Sci. 27, 305 (1997).

${ }^{2}$ D. K. Yang and S. T. Wu, Fundamentals of Liquid Crystal Devices (Wiley, New Jersey, 2006).

${ }^{3}$ M. Schadt, Jpn. J. Appl. Phys., Part 1 48, 03 B001 (2009).

${ }^{4}$ A. K. Bhowmik, Z. Li, and P. J. Bos, Mobile Displays: Technology and Applications (Wiley, England, 2008).

${ }^{5}$ M. Oh-e and K. Kondo, Appl. Phys. Lett. 67, 3895 (1995).

${ }^{6}$ Z. Ge, X. Zhu, T. X. Wu, and S.-T. Wu, J. Disp. Technol. 2 , 114 (2006).

${ }^{7}$ H. Hong, H. Shin, and I. Chung, J. Disp. Technol. 3, 361 (2007).

${ }^{8}$ S. H. Lee, S. L. Lee, and H. Y. Kim, Appl. Phys. Lett. 73, 2881 (1998).

${ }^{9}$ Y. Chen, Z. Y. Luo, F. L. Peng, and S. T. Wu, J. Disp. Technol. 9, 74 (2013).

${ }^{10}$ D. H. Kim, Y. J. Lim, D. E. Kim, H. Ren, S. H. Ahn, and S. H. Lee, J. Info. Disp. 15, 99 (2014).

${ }^{11}$ Z. Luo, D. Xu, and S. T. Wu, J. Disp. Technol. 10, 526 (2014).

${ }^{12}$ H. Chen, F. Peng, Z. Luo, D. Xu, S. T. Wu, M. C. Li, S. L. Lee, and W. C. Tsai, Opt. Mater. Express 4, 2262 (2014).

${ }^{13}$ K. H. Kim, H. Y. Kim, Y. H. Jeong, S. Y. Kim, Y. J. Lim, and J. Y. Lee, SID Int. Symp. Dig. Tech. Pap. 37, 755 (2006).

${ }^{14}$ Y. Momoi, K. Tamai, K. Furuta, T. R. Lee, K. J. Kim, C. H. Oh, and T. Koda, J. Soc. Info. Disp. 18, 134 (2010). 
${ }^{15}$ T. Tsuruma, Y. Goto, A. Higashi, M. Watanabe, H. Yamaguchi, and T. Tomooka, Proc. Eurodisplay 11, 13 (2011).

${ }^{16}$ R. Hatsumi, S. Fukai, Y. Kubota, A. Yamashita, M. Jikumaru, H. Baba, K. Moriya, D. Kubota, K. Kusunoki, Y. Hirakata, J. Koyama, S. Yamazaki, Y. Chubachi, and C. Fujiwara, J. Soc. Info. Disp. 21, 442 (2013).

${ }^{17}$ S. H. Perlmutter, D. Doroski, and G. Moddel, Appl. Phys. Lett. 69, 1182 (1996).

${ }^{18}$ Y. Yasuda, H. Naito, M. Okuda, and A. Sugimura, Mol. Cryst. Liq. Cryst. 263, 559 (1995).

${ }^{19}$ A. Sawada, K. Tarumi, and S. Naemura, Jpn. J. Appl. Phys., Part 1 38, 1418 (1999).

${ }^{20}$ G. Barbero, A. K. Zvezdin, and L. R. Evangelista, Phys. Rev. E 59, 1846 (1999).

${ }^{21}$ Y. Tanaka, Y. Goto, and Y. Iimura, Jpn. J. Appl. Phys., Part 2 38, L1115 (1999).

${ }^{22}$ S. Naemura and A. Sawada, Mol. Cryst. Liq. Cryst. 346, 155 (2000).

${ }^{23}$ H. De Vleeschouwer, A. Verschueren, F. Bougrioua, K. Neyts, G. Stojmenovik, S. Vermael, and H. Pauwels, Jpn. J. Appl. Phys., Part 1 41, 1489 (2002).

${ }^{24}$ M. Mizusaki, T. Miyashita, T. Uchida, Y. Yamada, Y. Ishii, and S. Mizushima, J. Appl. Phys. 102, 014904 (2007).

${ }^{25}$ D. Cristaldi, S. Pennisi, and F. Pulvirenti, Liquid Crystal Display Drivers (Springer, Netherlands, 2008).

${ }^{26}$ R. B. Meyer, Phys. Rev. Lett. 22, 918 (1969).

${ }^{27}$ W. Helfrich, Mol. Cryst. Liq. Cryst. 26, 1 (1974).

${ }^{28}$ I. H. Jeong, I. W. Jang, D. H. Kim, J. S. Han, B. V. Kumar, and S. H. Lee, SID Int. Symp. Dig. Tech. Pap. 44, 1368 (2013).
${ }^{29}$ S. Murakami and H. Naito, Jpn. J. Appl. Phys., Part 1 36, 773 (1997).

${ }^{30}$ H. D. Vleeschouwera, B. Verweirea, K. D'havea, and H. Zhang, Mol. Cryst. Liq. Cryst. 331, 567 (1999).

${ }^{31}$ H. Naito, M. Okuda, and A. Sugimura, Phys. Rev. A 44, R3434 (1991).

${ }^{32}$ A. Sugimura, N. Matsui, Y. Takahashi, H. Sonomura, H. Naito, and M. Okuda, Phys. Rev. B 43, 8272 (1991).

${ }^{33}$ V. G. Chigrinov, V. M. Kozenkov, and H. S. Kwok, Photoalignment of Liquid Crystalline Materials: Physics and Applications (Wiley, England, 2008).

${ }^{34}$ M. Mizusaki, T. Miyashita, and T. Uchida, J. Appl. Phys. 112, 044510 (2012).

${ }^{35}$ L. Lu, A. K. Bhowmik, and P. J. Bos, Liq. Cryst. 40, 7 (2013).

${ }^{36}$ L. R. Evangelista and G. Barbero, Phys. Rev. E 64, 021101 (2001).

${ }^{37}$ J. M. Seddon and J. D. Gale, Thermodynamics and Statistical Mechanics (Royal Society of Chemistry, Cambridge, 2001).

${ }^{38}$ S. Naemura and A. Sawada, Mol. Cryst. Liq. Cryst. 400, 79-96 (2003).

${ }^{39}$ H. Y. Hung, C. W. Lu, C. Y. Lee, C. S. Hsu, and Y. Z. Hsieh, Anal. Methods 4, 3631-3637 (2012).

${ }^{40}$ H. Seiberle and M. Schadt, Mol. Cryst. Liq. Cryst. 239, 229-244 (1994).

${ }^{41}$ H. Naito, Y. Yasuda, and A. Sugimura, Mol. Cryst. Liq. Cryst. 301, 85-90 (1997).

${ }^{42}$ M. Bremer, S. Naemura, and K. Tarumi, Jpn. J. Appl. Phys., Part 2 37, L88-L90 (1998).

${ }^{43}$ D. H. Kim, D. E. Kim, J. H. Lee, K. H. Song, S. H. Ahn, and S. H. Lee, SID Int. Symp. Dig. Tech. Pap. 45, 1421-1423 (2014).

${ }^{44}$ J. N. Israelachvili, Intermolecular and Surface Forces, 3rd Ed. (Academic Press, Oxford, 2011), p. 62. 